\title{
INTEGRATED DYNAMIC AND SIMULATION MODEL ON COUPLED CLOSED-LOOP WORKSTATION CAPACITY CONTROLS IN A MULTI-WORKSTATION PRODUCTION SYSTEM
}

\author{
Tao $\mathrm{Wu}$ \\ Leyuan Shi \\ Benjamin Quirt
}

Department of Industrial \& Systems Engineering University of Wisconsin-Madison

1550 Engineering Drive, Madison, WI 53706, USA

\begin{abstract}
In this paper, a dynamic model coupled with a simulation model is introduced to control a multi-workstation production system such that a given performance measure is achieved. In particular, we consider closed loop capacity controls for regulating WIP (Work-in-Process) at individual workstation. The capacity adjustments are consisted of both compensation for local disturbances and predictive control of downstream effects of capacity adjustments make up-stream in the system. Using real data collected from an industrial production system, we are able to demonstrate that our hybrid dynamic and simulation framework can effectively predict lead times associated with each workstation and thus to correctly plan production using a static capacity control system.
\end{abstract}

\section{INTRODUCTION}

Disturbances due to the dynamic nature of a production system can be attributed to many factors, ranging from target setting to fulfillment to production data acquisition to logistic performance measurement (Kim and Duffie, 2006). While disturbances are main factors causing deviations of WIP of the production system from the planned WIP. In order to prevent and correct the effects from disturbances, the Production Planning and Control (PPC) system can be implemented. However, the Production Planning and Control (PPC) system itself can be the source of disturbances in the system, so understanding how the system controls production and how this relates to the dynamic nature of production can lead to improved planning techniques (Kim and Duffie 2005). Additionally, the upstream disturbances have an effect on downstream operation for long periods of time when there is no communication between the production networks and this is amplified when reaction to capacity changes is limited (Kim and Duffie 2006). Therefore, coupled closed-loop capacity control polices are necessary applied to effectively control WIP in
N.A. Duffie

Department of Mechanical Engineering University of Wisconsin-Madison

1550 Engineering Drive, Madison, WI 53706, USA

to the planned WIP levels by accurately predicting correct reallocation of capacity of downstream workstation.

Some previous researches have been conducted on analyzing performance of closed-loop workstation capacity controls in a multi-workstation production system. Kim and Duffie (2006) propose a control-theoretic system architecture, in which control-theoretic methods are used to design coupling mechanisms and improve understanding and confidence in fundamental dynamic behavior by adding coupling at the control level combined with intrinsic coupling at the orderflow levels. They claim that system performance can be improved through control coupling between workstations. Towill and Cheema (1997) propose a closed-loop PPC concept with adaptive inventory control in decision support systems within in a multi-product medical supplies market.

This paper presents a dynamic model of a multiworkstation production system, then shows how the dynamic model of control is incorporated into a discrete event simulation model. Then a 5-workstation production system example as a case study is presented, by which the fundamental properties and dynamic behavior of the system are analyzed. Finally, based on results of the case study conclusions about the simulation are presented.

\section{DYNAMIC MODEL OF MULTIPLE WORK SYSTEMS}

Figure 2 shows the external inputs and outputs of the nworkstation production system. The external input and output rates represent orders flowing from and to the 0th workstation respectively. The planned capacity, $c_{p}(t)_{k}$, and planned WIP, wip $_{p}(t)_{k}$, for each of the $\mathrm{n}$ workstations is constant and unaffected by the production network. The input rate, $I(t)_{0}$, is the rate at which external orders flow into the production system.

Figure 1 illustrates the closed-loop and coupled capacity control mechanisms for the kth workstation in an $\mathrm{n}$-workstation production system. The input rate of a single 


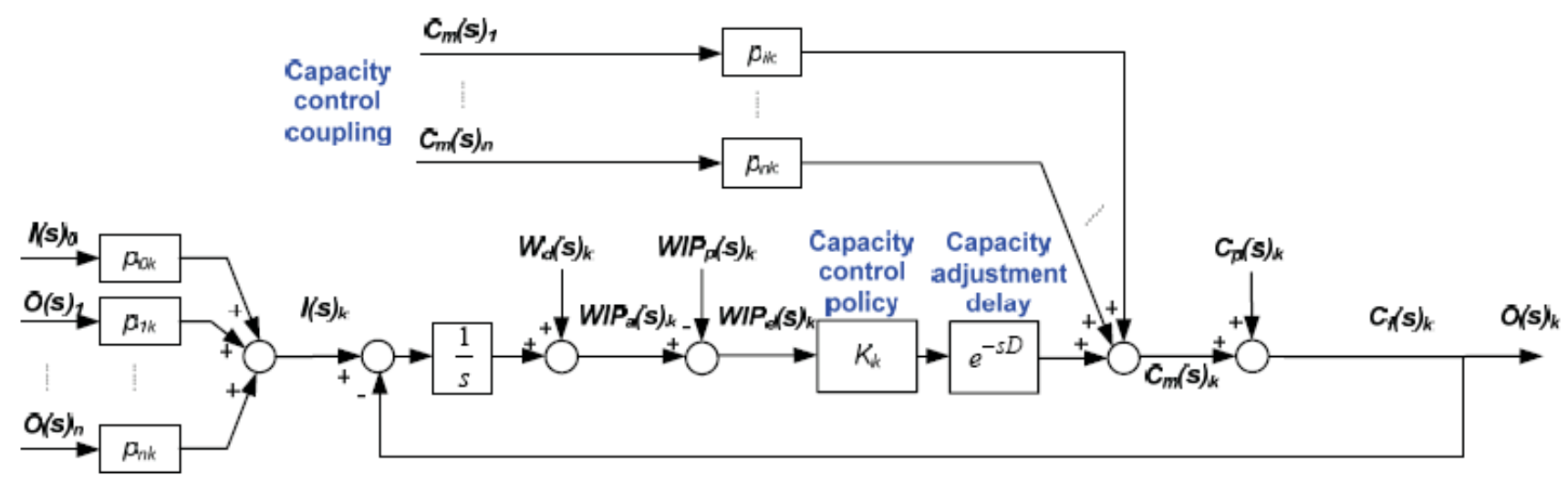

Figure 1: Closed-loop and coupled capacity control of the kth workstation.

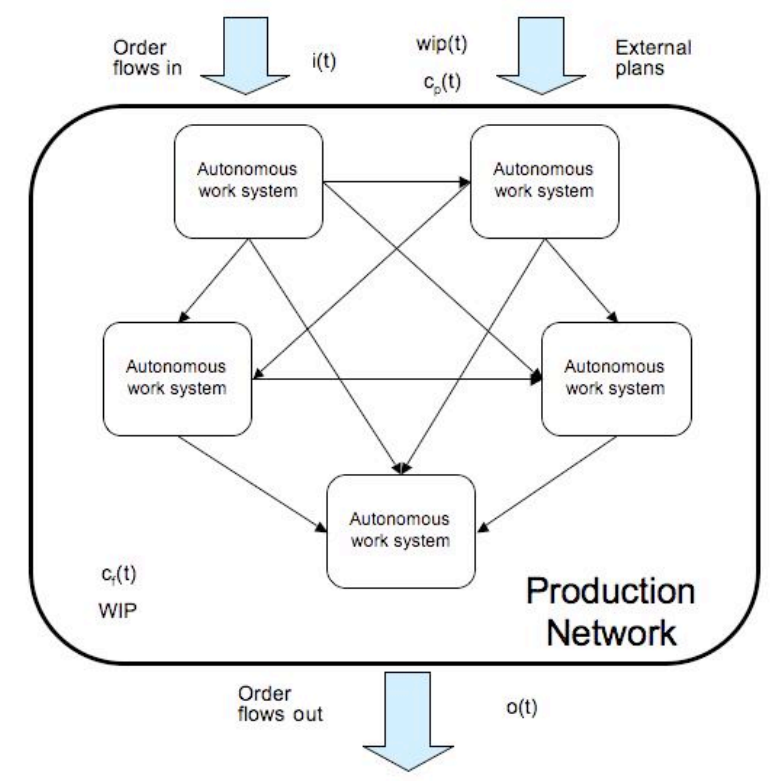

Figure 2: Production network of autonomous work system.

workstation is:

$$
I(t)_{k}=p_{0 k} I(t)_{0}+\sum_{i=1}^{n} p_{i k} O(t)_{i}
$$

where the fraction of orders going to workstation $\mathrm{k}$ is $p_{0 k}$. The fraction of orders going to workstation $\mathrm{i}$ to $\mathrm{k}$ from previous workstation is represented by matrix $p_{i k}$, and $O(t)_{i}$ is the output rate of the ith workstation (Wiendahl and Breithaupt 2000). Assuming the model is in a state where capacity is not effected by WIP, the output rate is assumed to be $c_{f}(t)_{k}$ :

$$
O(t)_{k} \approx c_{f}(t)_{k}
$$

The WIP in the system can be determined by taking the collective difference of output and input rates. Accounting for work disturbances, $W_{d}(t)_{k}$, the actual WIP, wip $p_{a}(t)_{k}$ in the system is:

$$
\text { wip }_{a}(t)_{k}=\int\left(I(t)_{k}-O(t)_{k}\right) d t+W_{d}(t)_{k}
$$

Maintaining level of wip $_{a}(t)_{k}$ equal to the planned levels will result in the planned result of eliminating the wip error, $\operatorname{wip}_{e}(t)_{k}$.

$$
\operatorname{wip}_{e}(t)_{k}=\operatorname{wip}_{a}(t)_{k}-\operatorname{wip}_{p}(t)_{k}
$$

The capacity adjustment and the speed of which wip $e_{e}(t)_{k}$ is corrected, is dependent upon the control policy gain $k_{c} s c d^{-1}$ and adjustments in upstream workstation capacity resulting in a capacity adjustment of:

$$
c_{m}(t)_{k}=k_{c} \text { wip }_{e}(t-D)_{k}+\sum_{i=1}^{n} p_{i k} c_{m}(t)_{i}
$$

The value of $k_{c}$ is determined based on the capacity control policy and $\mathrm{D}$ is the elapsed time necessary to change capacity at the workstation, assuming instantaneous change in capacity is not achievable. It is also assumed all workstations experience the same $k_{c}$ and D values. The full capacity of the kth workstation is:

$$
c_{f}(t)_{k}=c_{m}(t)_{k}+c_{p}(t)_{k}
$$


Wu, Shi, Quirt, and Duffie

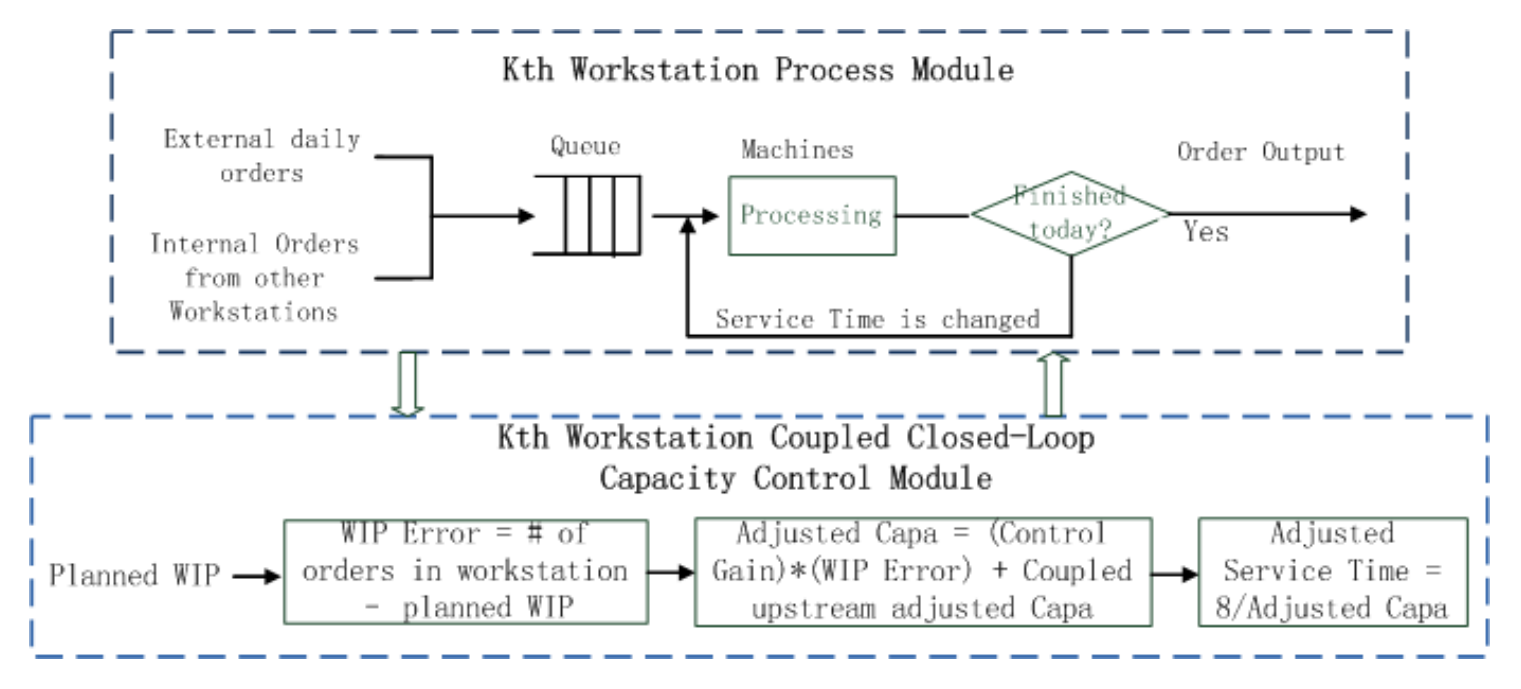

Figure 3: Discrete-event simulation model illustration of kth workstation.

\section{A DISCRETE EVENT SIMULATION MODEL FOR CAPACITY CONTROLS}

Based on the dynamic model, Kim and Duffie (2006) propose a control-theoretic method exploring its fundamental dynamic behavior. While we will present a discrete event simulation model to deal with this in the paper. Using Arena the discrete event simulation model(DES) of a multiworkstation production system is modeled.

Figure 3 shows the kth workstation simulation model illustration, in which there are two modules: one is the workstation process module, the other is the coupled closedloop capacity control module. The simulation modules for other workstations are similar to those of the kth workstation. In the simulation model, we set the run time to 8 hours for per shop calendar day (scd) and assume that the working time is from 0 to 8 o'clock of each day. When we calculate lead time for orders, the neglected 16 hours for each day will be counted on again. We assume that the order arrival time for each calendar day is at 0 o'clock and the WIP control logic is executed at $0 o^{\prime}$ clock daily. Regards to capacity adjustment, the corresponding service time adjustment is reflected, an average service time of an order for the kth workstation, $S t(t)_{k}$ can be calculated as follows:

$$
S t(t)_{k}=\frac{8}{c_{p}(t)_{k}+k_{c} w i p_{e}(t-D)_{k}+\sum_{i=1}^{n} p_{i k} c_{m}(t)_{i}}
$$

If the adjusted capacity is 0 or negative, the corresponding service time of orders is set to be infinite. On the following day the capacity adjustment is recalculated and the service time is set to a different value. If an order enters the workstation at the simulation time, $T_{\text {now }}$, and the simulation day, $T_{d a y}$, such that the service time exceeds the amount of available time left in the day then only a fraction of the order is processed. The fraction of work completed, $F r(t)_{k j}$, is then:

$$
F r(t)_{k j}=\frac{S t(t)_{k}-\left(8 * T_{\text {day }}-T_{\text {now }}\right)}{S t(t)_{k}}
$$

Therefore on the following day the service time of the unfinished job will be:

$$
S t(t+1)_{k j}=\left(1-F r(t)_{k j}\right) S t(t+1)_{k}
$$

We may give an example of explain how we handle the capacity adjustment, let us assume the capacity today 4 (orders/scd). The processing time is then 2 hours for each order, if an order input into the machine (or process module) at the 7th hour of an 8 hour workday, half of the work required is completed $\left(\operatorname{Fr}(t)_{k j}=0.5\right)$, leaving half for the following day. Let us assume the capacity for the following day is 8 (orders/scd), then processing time is 1 hour. Using equation 9 , the order will then require 0.5 hours of processing.

\section{A 5-WORKSTATION SYSTEM CASE STUDY}

\subsection{Real Industrial Order Flow Data}

The following example is based on production data from a metal forming company that supplies parts to the automotive industry. The behavior of the system is modeled using discrete capacity control model simulation. The data includes all orders, 659, entering the production system from scd 162 to scd 347 in 2001. For this analysis, the workstations are grouped into five workstations. 
Table 1: Summary of 5 workstations

\begin{tabular}{ccc}
\hline Workstation & Description & Number of Machines \\
\hline 1 & Shearing/Sawing & 6 \\
2 & Ring Rolling & 16 \\
3 & Drop Forging & 10 \\
4 & Heat Treatment & 8 \\
4 & Quality Control & 24 \\
\hline
\end{tabular}

Figure 4 shows the fraction of orders flowing from workstation $\mathrm{i}$ to workstation $\mathrm{j}$, in the form of matrix $p_{i j}$.

\begin{tabular}{|c|c|c|c|c|c|c|}
\hline $\begin{array}{c}\text { To } \\
\text { From }\end{array}$ & 0 & 1 & 2 & 3 & 4 & 5 \\
\hline 0 & 0 & $341 / 659$ & $295 / 659$ & $1 / 659$ & $7 / 659$ & $15 / 659$ \\
\hline 1 & 0 & 0 & $106 / 341$ & $235 / 341$ & 0 & 0 \\
\hline 2 & $9 / 401$ & 0 & 0 & 0 & $188 / 401$ & $204 / 401$ \\
\hline 3 & $7 / 236$ & 0 & 0 & 0 & $100 / 236$ & $129 / 236$ \\
\hline 4 & $27 / 295$ & 0 & 0 & 0 & 0 & $268 / 295$ \\
\hline 5 & $616 / 616$ & 0 & 0 & 0 & 0 & 0 \\
\hline
\end{tabular}

Figure 4: Order flow matrix $p_{i j}$.

And the planned capacity and WIP calculated from the data are shown in Figure 5.

\begin{tabular}{|c|c|c|c|c|}
\hline \multirow{2}{*}{ WS } & \multicolumn{3}{|c|}{$\mathrm{C}_{\mathrm{p}}$ [orders/scd] } & \multirow{2}{*}{$\begin{array}{c}\text { WIP } \\
\text { [orders] }\end{array}$} \\
\cline { 2 - 4 } & Weekday & Saturday & Sunday & 21.07 \\
\hline 1 & 4.72 & 0.92 & 0 & 18.92 \\
\hline 2 & 5.34 & 1.50 & 0 & 14.46 \\
\hline 3 & 2.95 & 0.42 & 0 & 14.87 \\
\hline 4 & 2.70 & 2.50 & 1.92 & 72.11 \\
\hline 5 & 6.28 & 0.83 & 0.08 & \\
\hline
\end{tabular}

Figure 5: Planned capacity and WIP.

\subsection{Simulation Results to Industrial Data}

In order to illustrate the dynamic behavior of multiworkstation production systems better, the input data are modified to eliminate weekends, the modified planned capacity is listed in Table 2. In this simulation model, we set the control gain $K_{c}$ to be 0.25 and set the delayed calender day, D, to be 1 . Table 3 shows the simulated results on actual WIP and lead time, which are calculated by eliminating data of the warm-up and shutdown states. The low standard deviation implies the simulation model effectively controls the actual WIP into the planned WIP levels. Lead times also exhibit low variation. In addition, Figures 6-10 show the simulated wipa $(t)_{k}$ levels for each of the 5 workstations.

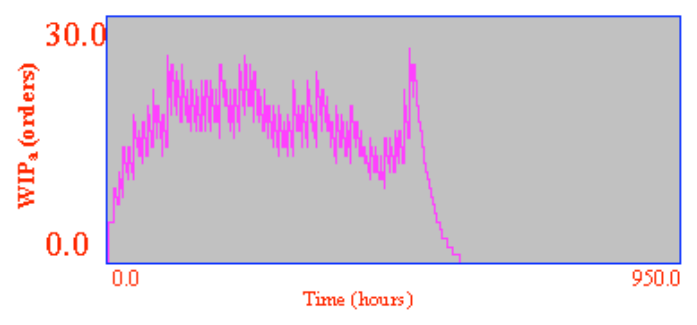

Figure 6: Workstation 1: Shearing \& Sawing.

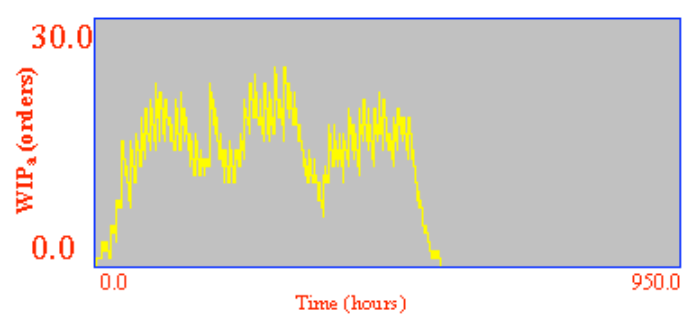

Figure 7: Workstation 2: Ring Rolling.

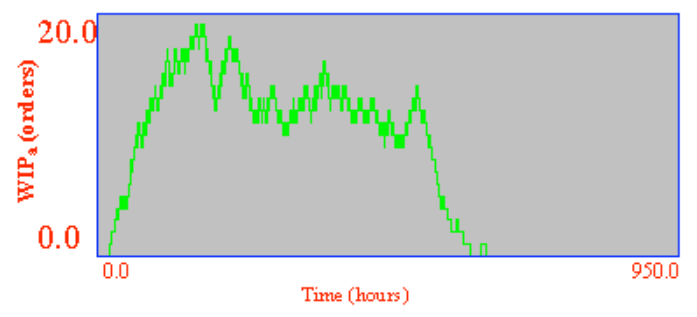

Figure 8: Workstation 3: Drop Forging.

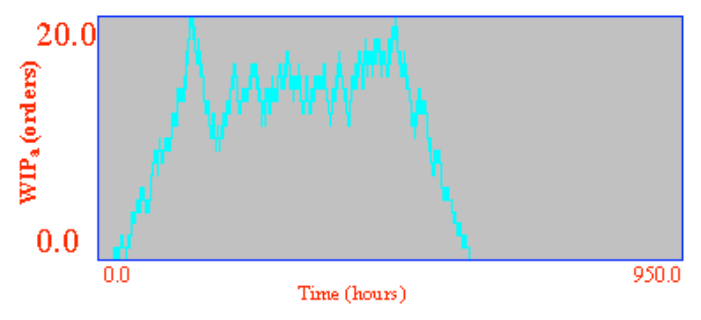

Figure 9: Workstation 4: Heat Treatment.

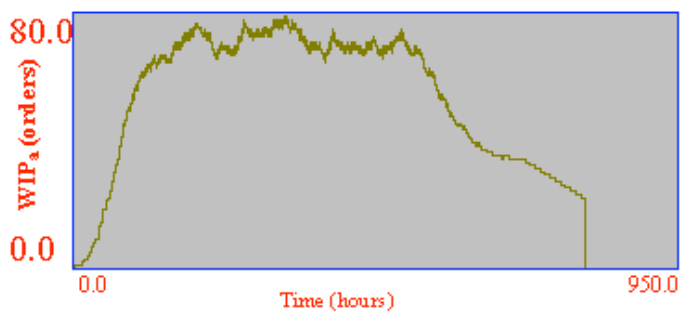

Figure 10: Workstation 5: Quality Control. 
Wu, Shi, Quirt, and Duffie

Table 2: The modified planned capacity after eliminating weekends

\begin{tabular}{cccccc}
\hline & W1 & W2 & W3 & W4 & W5 \\
\hline MPC & 5.32 & 6.27 & 3.69 & 4.60 & 9.60 \\
\hline
\end{tabular}

*MPC indicates modified planned capacity.

Table 3: Actual WIP and lead time obtained by simulation model

\begin{tabular}{ccccc}
\hline Planned WIP & \multicolumn{2}{c}{ SWIP[orders] } & \multicolumn{2}{c}{ Lead Time[scd] } \\
\hline & Mean & Deviation & Mean & Deviation \\
\hline 21.07 & 20.47 & 3.09 & 3.11 & 0.40 \\
18.92 & 18.45 & 3.30 & 2.36 & 0.42 \\
14.46 & 14.09 & 2.36 & 3.88 & 0.40 \\
14.87 & 14.65 & 2.90 & 3.20 & 0.46 \\
72.11 & 70.66 & 3.79 & 7.56 & 0.44 \\
\hline
\end{tabular}

*SWIP indicates the actual WIP result obtained by DES model.

\section{CONCLUSION}

An integrated dynamic and simulation model is introduced for multi-workstation production systems with coupled closed-loop capacity controls for regulating WIP in individual workstation, in which capacity adjustments is consisted of both compensation for local disturbances and predictive control of downstream effects of capacity adjustments made upstream in the system. The model is used to analyze the fundamental properties and dynamic behavior of multi-workstation production systems, which is illustrated using real data from an industrial production system. The simulation results show that the integration of the dynamic and simulation model is able to effectively correct the effects of work planning disturbances. The low variation of the lead time at each workstation indicates simulation is able to predict lead times based on real production data. The predicted lead time can be used to plan production in common static capacity planning systems used in industry, such as MRP.

\section{ACKNOWLEDGEMENTS}

This research was supported in part by the National Science Foundation under grant DMI-0646697 and by the Air Force of Scientific Research under grant FA9550-07-1-0390.

\section{REFERENCES}

Cheng, L., E. Subrahmanian, and A. Westerberg. 2004. Multi-Objective Decisions of Capacity Planning and
Production Uncertainty. Industrial and Engineering Chemistry Research 43 (9):2192-2208.

Duffie, N. and Roy, D. 2007. Dynamic Modeling of Production Networks of Autonomous Work Systems With Local Capacity Control.

Kim, J.H., and N.A. Duffie. 2006. Performance of Coupled Closed-Loop Capacity Controls in a Multi-Workstation Production System. Annals of the CIRP 55 (1):449-452.

Kim, J.H., and N.A. Duffie. 2005. Design and Analysis of Closed-Loop Capacity Control for a Multi-Workstation Production System. Annals of the CIRP 54 (1):455-458.

Ma, Y.H., and Y. Koren. 2004. Operation of Manufacturing Systems With Work-In-Process Inventory Control and Production. Annals of the CIRP 53 (1):361-365.

Pandey, P. 1997. Impact of Capacity Planning on Inventory in a Production System. International Journal of Computer Applications in Technology 10 (1-2):62-72.

Schneider, M., and H.P. Wiendahl. 2003. Logistic Measurement of Manufacturing Departments by the Use of Logistic Process Operating Curves (LPOCs). Germany: University of Hanover.

Segerstedt, A. 1996. A capacity-Constrained Multi-Level Inventory and Production Control Problem. Interational Journal of Production Economics 45 (1-3):449-461.

Towill, D., and P. Cheema. 1997. Analysis and Design of an Adaptive Minimum Reasonable Inventory Control. Production Planning and Control 8 (6):545-557.

Van, O.H., and J. Bertrand. 2003. The Effects of a Simple Arrival Rate Control Policy on Throughput and Work-In-Process in Production Systems With Workload Dependent Processing Rate. International Journal of Production Economics 85 (1):65-68.

Wiendahl, H.P., and J.W. Breithaupt. 2000. Automatic Production Control Applying Control Theory. International Journal of Production Economics 63 (1):33-46.

\section{AUTHOR BIOGRAPHIES}

TAO WU is a Ph.D student with Department of Industrial and Systems Engineering at University of WisconsinMadison. He received his M.S in industrial engineering from University of Wisconsin-Madison in 2007. Tao's research is devoted to develop the theory and applications of large-scale optimization algorithms of production planning and scheduling systems

LEYUAN SHI is a Professor with Department of Industrial and Systems Engineering at University of WisconsinMadison. She received her Ph.D. in Applied Mathematics from Harvard University in 1992, her M.S. in Engineering from Harvard University in 1990, her M.S. in Applied Mathematics from Tsinghua University in 1985, and her B.S. in Mathematics from Nanjing Normal University in 1982. Dr. Shi has been involved in undergraduate and 
graduate teaching, as well as research and professional service. Dr. Shis research is devoted to the theory and applications of large-scale optimization algorithms, discrete event simulation and modeling and analysis of discrete dynamic systems. She has published many papers in these areas. Her work has appeared in Discrete Event Dynamic Systems, Operations Research, Management Science, IEEE Trans., and, IIE Trans. She is currently a member of the editorial board for Journal of Manufacturing \& Service Operations Management, and is an Associate Editor of Journal of Discrete Event Dynamic Systems. Dr. Shi is a member of IEEE and INFORMS.

BENJAMIN QUIRT is a senior undergraduate student with Department of Industrial and Systems Engineering at University of Wisconsin-Madison.

N.A. DUFFIE is a Professor with Department of Mechanical Engineering at University of Wisconsin-Madison. He received his Ph.D. in Mechanical Engineering from University of Wisconsin-Madison in 1980, his M.S. in Mechanical Engineering from University of Wisconsin-Madison in 1974, and his B.S. in Mechanical Engineering from University of Wisconsin-Madison in 1972. Professor Duffie's research in manufacturing systems involves integrating sensors, actuators, computers and data bases into advanced automated production systems. He has developed controls for self-guided inspection machines and welding robots, high-performance material handling systems, and automated finishing systems for mold and die production and rework. He is studying highly distributed, non-hierarchical system control architectures in hope of reducing cost and complexity in large-scale, computer-controlled manufacturing systems while increasing flexibility and fault tolerance. 